\title{
Echinococcus granulosus genotypes circulating in alpacas (Lama pacos) and pigs (Sus scrofa) from an endemic region in Peru
}

\author{
Elizabeth Sánchez ${ }^{1,5 /+}$, Omar Cáceres², César Náquiraa ${ }^{1,3}$, \\ Eduardo Miranda', Franklyn Samudio ${ }^{4,5}$, Octavio Fernandes ${ }^{5}$ \\ 'Laboratório de Zoonosis Parasitaria 2Laboratório de Biotecnología y Biologia Molecular, Instituto Nacional de Salud, Lima, Peru \\ ${ }^{3}$ Laboratório de Parasitología, Instituto de Medicina Tropical Daniel Alcides Carrion, Universidad Nacional Mayor de San Marcos, Lima, Peru \\ ${ }^{4}$ Laboratório de Parasitología, Instituto Conmemorativo Gorgas de Estúdio de la Salud, Panamá, Panamá \\ ${ }^{5}$ Laboratório Interdisciplinar de Pesquisas Médicas, Instituto Oswaldo Cruz-Fioruz, Rio de Janeiro, RJ, Brasil
}

The identification of the genotypes of Echinococcus granulosus present in livestock and wild animals within regions endemic for cystic echinococcosis (CE) is epidemiologically important. Individual strains display different biological characteristics that contribute to outbreaks of $C E$ and that must be taken into account in the design of intervention programs. In this study, samples of hydatid cysts due to E. granulosus were collected from alpacas (4) in Puno and pigs (8) in Ayacucho in Peru, an endemic region for CE. Polymerase chain reaction amplification and DNA sequencing of specific regions of the mitochondrial cytochrome C oxidase subunit 1 and NADH dehydrogenase subunit 1 genes confirmed the presence of a strain common to sheep, the G1 genotype, in alpacas. Two different strains of E. granulosus were identified in pigs: the G1 and the G7 genotypes. This is the first report of the G1 genotype of E. granulosus in alpacas in endemic regions of CE in Peru.

Key words: Echinococcus granulosus - genotype, alpaca - Peru

The metacestode of the tapeworm Echinococcus granulosus is responsible for cystic echinococcosis (CE) and can infect humans and several animal species. In Peru, CE is endemic in the central-southern regions of the country, mainly within the cattle-producing areas. The prevalence of human cases of CE in Peru is one of the highest in the world (Moro et al. 2011). Worldwide, E. granulosus displays intraspecific variations that permit its classification into 10 different strains or genotypes, which are associated with distinct intermediate host species (Bowles et al. 1992, Bowles \& McManus 1993, Tompson \& McManus 2002, Lavikainen et al. 2003). A previous study of $21 \mathrm{hu}-$ man cases of CE from Peru demonstrated the predominance of the G1 genotype, the strain common to sheep, with one case of the G6 genotype (Santivañez et al. 2008). The G1 genotype was also identified as the only genotype present in isolated from cattle, sheep and humans from endemic regions in Peru (Sanchez et al. 2010). In another endemic region of Peru, a study reported the presence of the G6 genotype (camel strain) in human and goats, the G7 genotype in pigs and the G1 genotype in sheep, cattle, pigs, goats and humans (Moro et al. 2009). The G7 pig strain has also been detected in cases of CE from the less endemic area of Lima, Peru (Moro et al. 2009).

The G7 genotype differs from other strains both morphologically and developmentally with regard to the maturation rate, epidemiology and genetics (Eck-

Financial support: CAPES (to ES)

+ Corresponding author: betesanchez@hotmail.com

Received 15 June 2011

Accepted 18 October 2011 ert \& Thompson 1997, Bowles \& McManus 1993). The presence of this strain has been previously described in pigs and humans in Europe, Russia, South America and Mexico (Kedra et al. 2000, Turceková et al. 2003, Haag et al. 2004, Varcasia et al. 2006, Villalobos et al. 2007, Schneider et al. 2010).

In this paper, we report the genotypes of E. granulosus circulating in alpacas and pigs in endemic regions for $\mathrm{CE}$ in Peru (Table). Alpacas and pigs represent economically important animals with consistent interactions with the local population. Following slaughter in abattoirs, hydatid cysts due to E. granulosus were collected from four alpacas and eight pigs. All of the cysts collected from alpacas were fertile. In pigs, all of the cysts from the lungs were fertile, but only half of the cysts from the liver were fertile (Table). The high percentage of fertile cysts in alpacas and pigs suggests a higher risk of transmission to other animals and humans in these regions.

Genotypes were determined by analysing two mitochondrial genes, cytochrome $\mathrm{C}$ oxidase subunit $1(\mathrm{CO} 1)$ and NADH dehydrogenase subunit 1 (ND1). DNA was isolated from each individual cyst sample according to the manufacturer's instructions (QIAamp DNA Mini Kit-QIAGEN, Germany). Fragments of the CO1 and ND1 genes were polymerase chain reaction (PCR)-amplified (Taq polymerase, Invitrogen). A partial region of the $\mathrm{CO} 1$ gene was amplified following a previously described protocol (Sánchez et al. 2010). The ND1 region was amplified using a unique primer set designed for this study: EL1F: 5'GTTTTTGGGTTAGTCTCTGG3' and EL1R: 5'ATCATAACGAACACGTGG3'. Briefly, 20 ng of DNA from $E$. granulosus was used in all reactions. The thermal profile of the PCR ND1 reaction was as follows: denaturation for $3 \mathrm{~min}$ at $95^{\circ} \mathrm{C}$, followed by 35 cycles of 30 sec at $95^{\circ} \mathrm{C}, 30 \mathrm{sec}$ at $55^{\circ} \mathrm{C}$ and $45 \mathrm{sec}$ at $72^{\circ} \mathrm{C}$ and a final 
incubation at $72^{\circ} \mathrm{C}$ for $7 \mathrm{~min}$ in a Gene Amp PCR System 9700 (Applied Biosystems). The PCR amplification of the CO1 and ND1 genes was successful in all isolates and generated products of approximately $450 \mathrm{bp}$ and $800 \mathrm{bp}$, respectively. The PCR products of the CO1 and ND1 genes were purified and sequenced using the BigDye Terminator v3.1 Cycle sequencing kit (Applied Biosystems) as reported previously (Otto et al. 2008).

Partial sequences of the CO1 (399 bp) and ND1 (756 bp) genes were successfully obtained for all samples. For strain identification, the sequences were aligned us- ing MEGA5 (megasoftware.net) and compared with the $\mathrm{CO} 1$ and ND1 reference sequences of the genotypes of $E$. granulosus present in GenBank (Fig. 1). The sequences of the isolates from the four alpacas and four of the pigs showed identity with the G1 genotype for both genes. The sequences of the other four isolates from pigs showed identity with the G7 genotype for both genes. The nucleotide sequence of the $\mathrm{CO} 1$ gene revealed that one isolate from an alpaca and three pig isolates had complete identity with the reference sequences of the G1 genotype (accessions U50464, GU233854 and GU233945). The

TABLE

Origins and characteristics of Peruvian isolates of Echinococcus granulosus

\begin{tabular}{lcccccc}
\hline Geographic origin & Host & Genotype & $\begin{array}{c}\text { Isolates } \\
\text { (n) }\end{array}$ & Hydatid cyst localization & \multicolumn{2}{c}{$\begin{array}{c}\text { Fertility } \\
(\%)\end{array}$} \\
\hline Puno & Alpaca & G1 & 4 & Lung & Total \\
Ayacucho & Pig & G1 & 4 & Lung & 100 & 4 \\
& Pig & G7 & 4 & Liver & 100 & 4 \\
\hline
\end{tabular}

$\mathrm{CO1}$

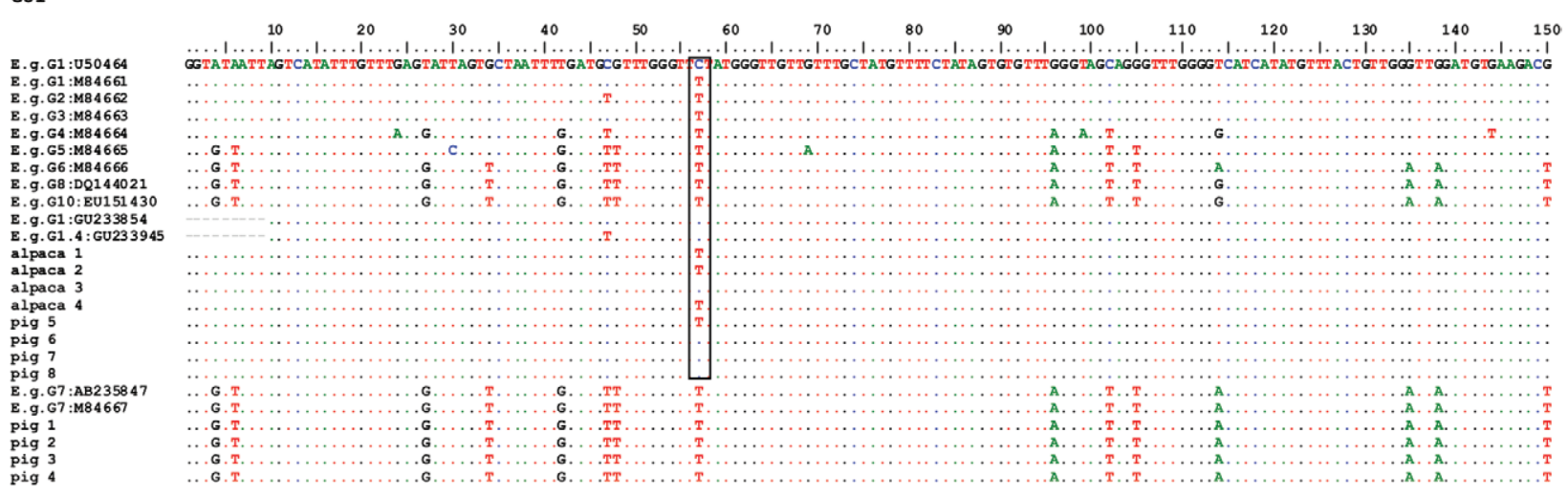

ND1

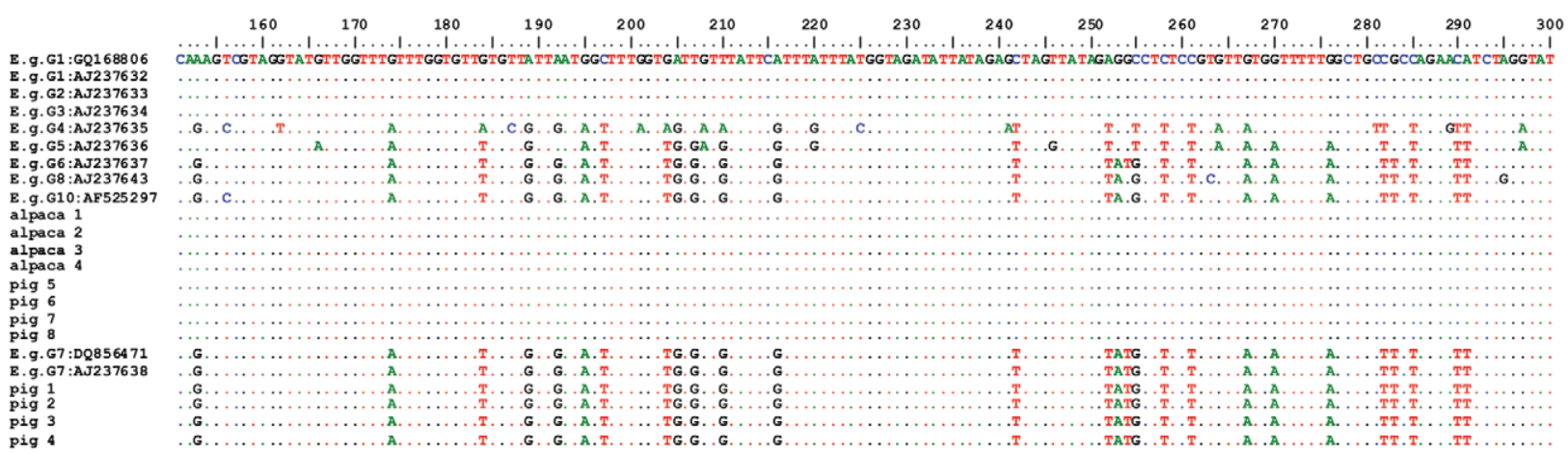

Fig. 1: nucleotide sequences of partial regions of the cytochrome C oxidase subunit 1 (CO1) (399 bp) and NADH dehydrogenase subunit 1 (ND1) (756 bp) genes for 12 Peruvian isolates of Echinococcus granulosus aligned with reference sequences deposited in GenBank. All sequences from alpaca and four from pigs are referenced to the G1 genotype; other four sequences from pig are referenced to the G7 genotype, for both mitochondrial genes. Deviations from the G1 sequence are displayed as individual nucleotides. The boxed region denotes a base pair that distinguishes the two reference sequences for G1. 
other three isolates from alpacas and the remaining G1 isolate from pigs showed complete identity with a different reference sequence of G1 (accession M84661). These G1 reference sequences and the isolates exhibit nucleotide polymorphisms at the 57 th position $(\mathrm{C} \rightarrow \mathrm{T})$ based on the numbering shown in Fig. 1. The sequences from this study were submitted to the GenBank database with the accessions JF828330-JF828341 for the CO1 gene and JF828342-JF828353 for the ND1 gene.

The analyses of these two mitochondrial marker genes for the E. granulosus isolates confirmed the existence of only the genotype G1 (common sheep strain) in all isolates from the studied alpacas. This is the first report of the G1 genotype of E. granulosus in alpacas from endemic regions of Peru. Two distinct genotypes, G1 and G7 (pig strain), were found among the isolates from pigs. The G1 genotype is the most common strain identified in humans and animals worldwide. In Peru, previous studies have demonstrated a predominance of the G1 genotype (common sheep strain) in humans and in hydatid cysts from sheep, cattle, goats and pigs in endemic regions (Santivañez et al. 2008, Moro et al. 2009, Sánchez et al. 2010). However, the G7 genotype (pig strain) has only been previously identified in pigs from Lima, a city considered to be a low endemic area for $E$. granulosus (Moro et al. 2009). The present study supplemented these findings by examining isolates from pigs collected from Ayacucho, an endemic region for $\mathrm{CE}$ in Peru. These findings also show that pigs can be infected by the G1 genotype or the G7 genotype in the region studied, confirming that pigs can be infected by multiple genotypes of E. granulosus (Eckert \& Thompson 1997). The G7 genotype has been previously described in human isolates in several countries around the world. In Peru, no reports describe the presence of genotype G7 in human cases of CE.

A phylogenetic tree of the $\mathrm{CO} 1$ and ND1 genotypes was constructed using the Maximum Likelihood algorithm with the Tamura-Nei distance, as the model of evolution (Fig. 2) (Tamura \& Nei 1993). Phylogenetic analysis showed two major clades. One larger clade included eight sequences grouped within the G1 genotype and the other included four sequences clustered with the G7 genotype, for both the CO1 and ND1 genes (Fig. 2). In addition, the clade grouping the G1 genotypes showed two subgroups: four sequences grouped with one G1 genotype (accession U50646) and other four sequences grouped with another G1 genotype (accession M84661). This grouping shows that there are two groups of G1 genotype strains present in the endemic regions of Peru.

In conclusion, our results indicate that more than one strain of E. granulosus circulates among the intermediate hosts of this parasite in endemic regions of Peru. It was also shown that pigs can be infected by both the G1 and G7 genotypes. Complementary studies will have to be performed to demonstrate the role of the pig strain in human disease in Peru. This knowledge will have important implications for the prevention and control of this zoonosis in Peru.
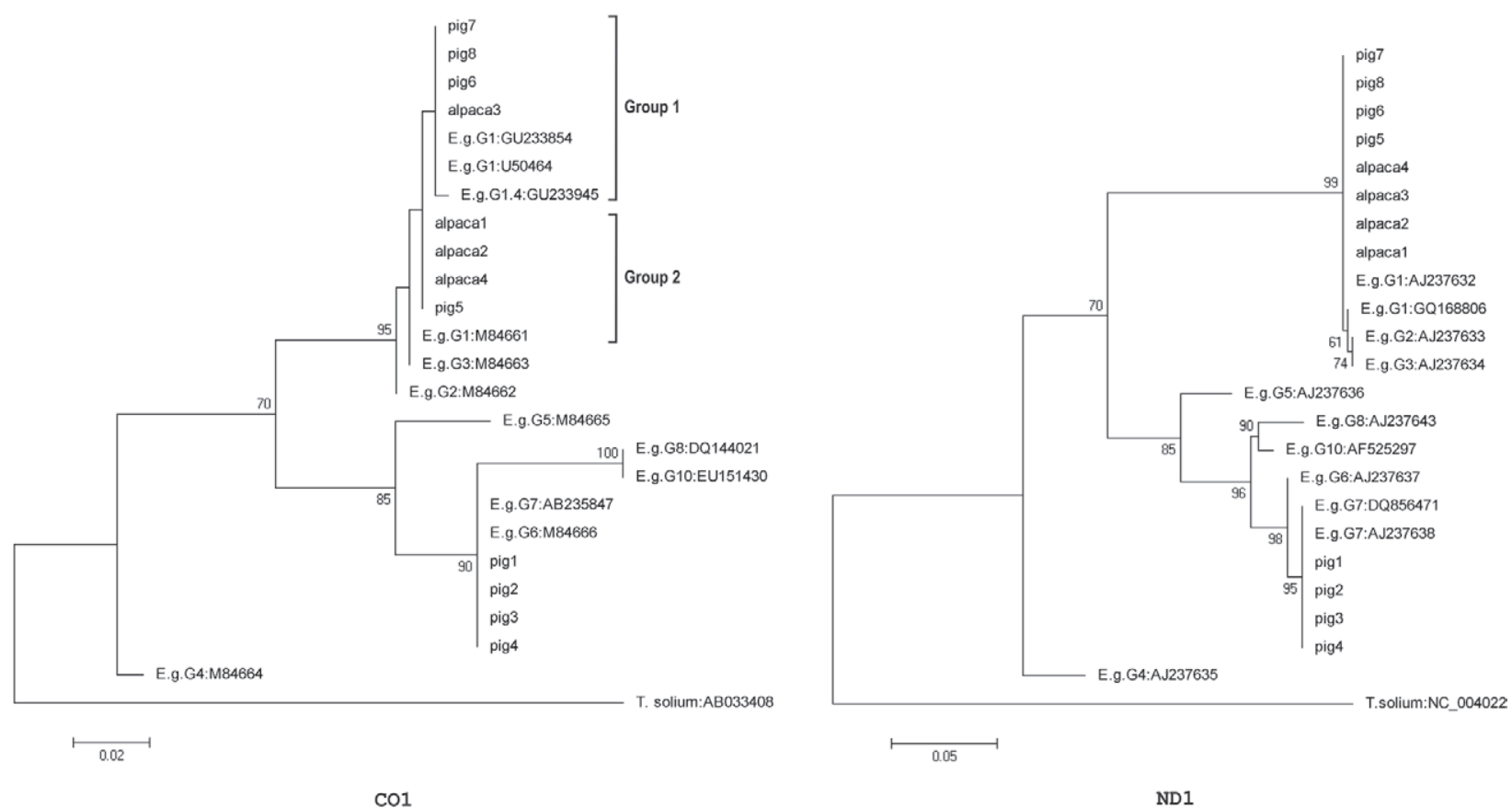

Fig. 2: phylogenetic tree obtained for Echinococcus granulosus isolates sequences in the present study. The trees were generated using Maximum Likelihood analysis based on an alignment of partial mitochondrial C oxidase subunit 1 (CO1) and NADH dehydrogenase subunit 1 (ND1) genes. The clade G1 genotypes showed Group 1 [4 sequences grouped with G1 genotype (accessions U50646, GU233854 and GU233845)] and Group 2 [4 sequences grouped by G1 genotype (accession M84661)].The numbers at the nodes indicate percentage bootstrap support obtained from 1,000 replications. A sequence from Taenia solium (AB033408 from CO1 and NC 004022 from ND1) was used as an outgroup to root the tree. 


\section{ACKNOWLEDGEMENTS}

To Herbet Larry, Regional Laboratory of Puno, for their help in samples collection, to Dr Adeilton Brandão, for their technical assistance, to Dr William Provance Jr, for the critical reading and English corrections of the paper, and to Heloísa Nogueira, for their help in designing the figures.

\section{REFERENCES}

Bowles J, Blair D, McManus DP 1992. Genetic variants within the genus Echinococcus identified by mitochondrial sequencing. Mol Biochem Parasitol 54: 65-174.

Bowles J, McManus DP 1993. NADH dehydrogenase 1 gene sequences compared for species and strains of the genus Echinococcus. Int J Parasitol 23: 969-972.

Eckert J, Thompson RCA 1997. Intraspecific variation of Echinococcus granulosus and related species with emphasis on their infectivity to humans. Acta Trop 64: 19-34.

Haag KL, Ayala FJ, Kamenetzky L, Gutierrez AM, Rosenzvit M 2004. Livestock trade history, geography and parasite strains: the mitochondrial genetic structure of Echinococcus granulosus in Argentina. J Parasitol 90: 234-239.

Kedra AH, Tkach VV, Swiderski Z, Pawlowski Z, Emets A, Pawlowski J 2000. Molecular characterization of Echinococcus granulosus from a wild boar. Acta Parasitol 45: 121-122.

Lavikainen A, Lehtinen MJ, Meri T, Hirvelä-Koski V, Meri S 2003. Molecular genetic characterization of the Fennoslandian cervid strain, a new genotypic group (G10) of Echinococcus granulosus. Parasitology 127: 207-215.

Moro PL, Budke CM, Schantz PM, Vasquez J, Santivañez SJ, Villavicencio J 2011. Economic impact of cystic echinococcus in Peru. PLoS Negl Trop Dis 5: el179.

Moro PL, Nakao M, Ito A, Shantz PM, Cavero C, Cabrera L 2009. Molecular identification of Echinococcus isolates from Peru. Parasitol Int 58: 184-186.
Otto TD, Vasconcellos EA, Gomes LHF, Moreira AS, Degrave WM, Mendonça-Lima L, Alves-Ferreira M 2008. ChromaPipe: a pipeline for analysis, quality control and management for a DNA sequencing facility. Genet Mol Res 7: 861-871.

Sánchez E, Cáceres O, Náquira C, Garcia D, Patiño G, Herrera S, Volotão AC, Fernandes O 2010. Molecular characterization of Echinococcus granulosus from Peru by sequencing of the mitochondrial cytochrome $\mathrm{C}$ oxidase subunit 1 gene. Mem Inst Oswaldo Cruz 105: 806-810.

Santivañes SJ, Gutierrez AM, Rosenzvit MC, Muzulin PM, Rodriguez ML, Vasquez JC, Rodriguez S, Gonzalez AE, Gilman RH, Garcia HH, The Cysticercosis Working Group in Peru 2008. Human hydatid disease in Peru is basically restricted to Echinococcus granulosus genotypes G1. Am J Med Hyg 79: 89-92.

Schneider R, Gollackner B, Schindl M, Tucek G, Auer H 2010. Echinococcus canadienses G7 (pig strain): an underestimated cause of cystic Echinococcus in Australia. Am J Med Hyg 82: 871-874.

Tamura K, Nei M 1993. Estimation of the number of nucleotide substitutions in the control region of the mitochondrial DNA in humans and chimpanzees. Mol Biol Evol 10: 512-526.

Thompson RCA, McManus DP 2002. Towards a taxonomic revision of the genus Echinococcus. Trends Parasitol 18: 452-457.

Turceková L, Snábel V, D’Amelio S, Busi M, Dubinský P 2003. Morphological and genetic characterization of Echinococcus granulosus in the Slovak Republic. Acta Trop 85: 223-229.

Varcasia A, Canu S, Lightowlers MW, Scala A, Garippa G 2006. Molecular characterization of Echinococcus granulosus strain in Sardinia. Parasitol Res 98: 273-277.

Villalobos N, González LM, Morales J, Aluja AS, Jiménez MI, Blanco MA, Harrison LJS, Parkhouse RME, Gárate T 2007. Molecular identification of Echinococcus granulosus genotypes (G1 and G7) isolated from pigs in Mexico. Vet Parasitol 147: 185-189. 